\title{
Monitoring multiple deadlines relies on spatial processing in posterior parietal cortex
}

\section{Zimmermann, Marius; Kubik, Veit; Persson, Jonas; Mäntylä, Timo}

Published in:

Journal of Cognitive Neuroscience

Link to article, DOI:

10.1162/jocn_a_01435

Publication date:

2019

Document Version

Publisher's PDF, also known as Version of record

Link back to DTU Orbit

\section{Citation $(A P A)$ :}

Zimmermann, M., Kubik, V., Persson, J., \& Mäntylä, T. (2019). Monitoring multiple deadlines relies on spatial processing in posterior parietal cortex. Journal of Cognitive Neuroscience, 31(10), 1468-1483.

https://doi.org/10.1162/jocn_a_01435

\section{General rights}

Copyright and moral rights for the publications made accessible in the public portal are retained by the authors and/or other copyright owners and it is a condition of accessing publications that users recognise and abide by the legal requirements associated with these rights.

- Users may download and print one copy of any publication from the public portal for the purpose of private study or research.

- You may not further distribute the material or use it for any profit-making activity or commercial gain

- You may freely distribute the URL identifying the publication in the public portal 


\title{
Monitoring Multiple Deadlines Relies on Spatial Processing in Posterior Parietal Cortex
}

\author{
Marius Zimmermann ${ }^{1,2}$, Veit Kubik ${ }^{1,3,4}$, Jonas Persson ${ }^{5,6}$, and Timo Mäntylä ${ }^{1}$
}

\begin{abstract}
Proactively coordinating one's actions is an important aspect of multitasking performance due to overlapping task sequences. In this study, we used fMRI to investigate neural mechanisms underlying monitoring of multiple overlapping task sequences. We tested the hypothesis that temporal control demands in multiple-task monitoring are offloaded onto spatial processes by representing patterns of temporal deadlines in spatial terms. Results showed that increased demands on time monitoring (i.e., responding to concurrent deadlines of one to four component tasks) increasingly activated regions in the left inferior parietal lobe and the precuneus. Moreover, independent measures of
\end{abstract}

\section{INTRODUCTION}

Modern life imposes increasing demands on our ability to keep track of multiple overlapping tasks that include various timelines and deadlines. For example, we may pay attention to TV programs or cell phone notifications while taking care of the household or work. During multitasking, several different tasks or instances of the same task are combined and executed in concert with overlapping timelines (Strobach, Wendt, \& Janczyk, 2018; Oswald, Hambrick, \& Jones, 2007; Burgess, Veitch, de Lacy Costello, \& Shallice, 2000). Previous behavioral (Hambrick, Oswald, Darowski, Rench, \& Brou, 2010; Konig, Buhner, \& Murling, 2005; Logie, Cocchini, Delia Sala, \& Baddeley, 2004; Meyer \& Kieras, 1997) and neuroimaging (Kübler \& Schubert, 2017; Tschernegg et al., 2017; Strobach, Antonenko, Schindler, Flöel, \& Schubert, 2016; Verghese, Garner, Mattingley, \& Dux, 2016; Hsu, Zanto, Anguera, Lin, \& Gazzaley, 2015; Medeiros-Ward, Watson, \& Strayer, 2015; Nijboer, Borst, van Rijn, \& Taatgen, 2014; Deprez et al., 2013; Stelzel, Kraft, Brandt, \& Schubert, 2008; Szameitat, Lepsien, von Cramon, Sterr, \& Schubert, 2006; Collette et al., 2005) studies indicate that executive functions play a critical role in multitasking. However, most studies typically

\footnotetext{
${ }^{1}$ Stockholm University, ${ }^{2}$ Technical University of Denmark, ${ }^{3}$ Humboldt University zu Berlin, ${ }^{4}$ Martin-Luther-University Halle-Wittenberg, ${ }^{5}$ Aging Research Center, Karolinska Institute \& Stockholm University, Stockholm, Sweden, ${ }^{6}$ Örebro University

spatial abilities correlated with multiple-task performance beyond the contribution of working memory. Together, these findings suggest that monitoring and coordination of temporally overlapping task timelines rely on cortical processes involved in spatial information processing. We suggest that the precuneus is involved in tracking of multiple task timelines, whereas the inferior parietal lobe constructs spatial representations of the temporal relations of these overlapping timelines. These findings are consistent with the spatial offloading hypothesis and add new insights into the neurocognitive mechanisms underlying the coordination of multiple tasks. concern isolated, experimentally tractable, aspects of dualand multitasking, using structured or bottom-up controlled paradigms (see also Logie, Trawley, \& Law, 2011).

In contrast, everyday multitasking usually involves planned (top-down controlled) task management (e.g., preparing breakfast or carrying out multiple errands; Logie et al., 2011; Craik \& Bialystok 2006), with high demands on time keeping (i.e., keeping track of overlapping task timelines and deadlines). Executive control demands are typically high in proactive tasks, but these accentuated control requirements can be alleviated by relying on prior knowledge and experience (Loukopoulos, Dismukes, \& Barshi, 2009; Wickens, 2008), as well as cognitive offloading strategies (Risko \& Gilbert, 2016). Specifically, studies in different domains of cognitive sciences have shown that humans can change the task requirements (and affordances) by relying both on internal and external representations of the task (Wilson \& Golonka, 2013; Zhang \& Norman, 1994; for an overview, see also Risko \& Gilbert, 2016).

Reflecting this "offloading" perspective, we have previously proposed a mechanism for alleviating control demands in multitasking performance. In detail, when task-specific knowledge is not available or insufficient, temporal control demands during multitasking are offloaded onto spatial abilities by representing patterns of temporal deadlines in spatial terms (Todorov, Del Missier, Konke, \& Mäntylä, 2015; Mäntylä, 2013). This idea resonates with similar views of "time-to-space mappings" in other domains of cognitive sciences, which suggest that temporal and spatial processing are closely related (e.g., Gijssels, Bottini, 
Rueschemeyer, \& Casasanto, 2013; Núñez \& Cooperrider, 2013; Bonato, Zorzi, \& Umiltà, 2012; Dehaene \& Brannon, 2011). This "spatial offloading hypothesis" is supported by several studies showing that spatial ability (i.e., mental rotation) and executive functioning (i.e., working memory updating) are independent predictors of multitasking performance and that spatial ability predicts multitasking over and beyond executive functioning (Todorov, Kubik, Carelli, Del Missier, \& Mäntylä, 2018; Mäntylä, Coni, Kubik, Todorov, \& Del Missier, 2017; Todorov, Del Missier, \& Mäntylä, 2014; Mäntylä, 2013; Morgan et al., 2013; Logie et al., 2011). Moreover, it was shown that multitasking, compared with dual tasking, involves an incremental contribution of spatial ability (Kubik, Zimmermann, Del Missier, Frick, \& Mäntylä, 2018, 2019) and that concurrent spatial load selectively impairs multitasking performance (Todorov et al., 2018; Mäntylä et al., 2017).

The aim of this study was to test a central assumption of the spatial offloading hypothesis, namely that spatial processes are involved when keeping track of multiple overlapping task timelines. Accordingly, we expected neural areas involved in spatial processing, especially superior and inferior parietal cortex (Sack, 2009; Zacks, 2008; Halari et al., 2006; Jordan, Heinze, Lutz, Kanowski, \& Jäncke, 2001; Kesner \& Long, 1998; Richter, Ugurbil, Georgopoulos, \& Kim, 1997; Cohen et al., 1996), and the hippocampus (Eichenbaum, 2017) to be incrementally engaged when the demands on time monitoring are increased using parametric analyses. Additionally, a modelbased approach was used to investigate whether the spatial offloading hypothesis is specific to multitasking situations (see Mäntylä, 2013) or generally valid for timemonitoring tasks in a wider range of situations (see also Kubik et al., 2018, 2019). Specifically, we tested whether recruitment of spatial processing areas increases gradually with time-monitoring demands (approximated by a linear increase model, reflecting general processes) or whether spatial processing areas are selectively recruited when the demands on time monitoring reach a threshold, for example, only during multitasking situations (i.e., following a step-like pattern).

\section{METHODS}

\section{Participants}

Twenty-four healthy, right-handed participants aged between 18 and 35 years $(M=27.9$ years, $S D=4.1$ years; 12 women) took part in the study. Participants were recruited from a large-scale behavioral study, in which they completed a series of cognitive tasks, including tests of mental rotation, working memory, and different versions of time-based monitoring tasks (see also Kubik et al., 2018, 2019). The behavioral study was completed approximately 6 months before the fMRI study. All participants were screened for claustrophobia, neurological and psychiatric medications, and MRI contraindication, and all had normal or corrected-to-normal vision using scanner-compatible glasses or contact lenses. One participant was excluded from the analysis due to excessive head movements $(>3 \mathrm{~mm})$ during all scanning sessions. Participants received financial compensation of 600 SEK for participation. The study was approved by the regional ethical review board in Stockholm, and written consent was obtained from all participants before the study commenced.

\section{Tasks}

\section{Countdown Task}

Monitoring of multiple timelines was tested with an adapted version of the "countertask" paradigm (Todorov et al., 2014; Mäntylä, 2013). In the original version, participants monitor a number of counters (clocks) and respond to predefined targets (e.g., Mäntylä, 2013). For the adapted version used here, forward-running counters of numbers were replaced with backward-running letter loops (letter countdowns) with fixed targets (Figure 1). Therefore, in the current task, inherent spatial processing was reduced by removing the need to update and maintain (numerical) target values and thus reducing possible spatial associations of the stimuli (further spatial associations due to stimulus-response mappings are controlled for using a control task; see below). This adapted task was recently shown to be highly correlated to and reveal similar associations with the cognitive reference measures (e.g., executive functioning, spatial ability) as the original counter task (Kubik et al., 2018, 2019). Time-monitoring demands were manipulated by varying the number of letter countdowns that had to be monitored and responded to. Specifically, the task included conditions with one to four letter countdowns at a time. Letter countdowns consisted of loops of letter series that followed the alphabet backward (e.g., H-G-F-E-...), until $A$, at which point (after a 2 -sec pause) the loop started again (here referred to as a single countdown series). The letter $A$ was defined as a target letter for each countdown series. Participants had to respond to the occurrence of the target letter as accurately as possible by pressing a "target button." Multiple countdown series formed a trial, during which a number of countdowns, varying from one to four (referred to as conditions), had to be monitored and responded to continuously for $60 \mathrm{sec}$. Importantly, letter countdowns were not continuously visible; instead, participants needed to uncover them by pressing one of four monitor buttons. This displayed the corresponding countdown for $1 \mathrm{sec}$, whereas no other countdowns could be displayed. Target button presses were assigned to the latest monitored countdown. Thus, participants had to make sure that, before indicating a target occurrence, the corresponding countdown was displayed last. The target button could, but did not have to, be pressed while the corresponding countdown was displayed. Upon pressing the target button, the monitored countdown changed into hidden state again, allowing for displaying of other 
Figure 1. Experimental task. (A) Timeline of a single countdown series and corresponding responses. Participants responded on two separate response boxes, one with four monitor buttons (right hand) and the other one with a single target button (left hand). The task is presented on a screen. The lower part of the screen contains information about the active countdowns (colored boxes). Countdowns follow the alphabet but are generally hidden from view. Letter countdowns were displayed in the upper part of the screen for 1 sec upon pressing of one of the four corresponding monitor buttons (Screen 2; green series displayed upon pressing of the green monitor button, at countdown state " $H$ "). After $1 \mathrm{sec}$, the countdown is covered again (third screen), until a monitor button is pressed again (fifth screen, " $A$ "). When the countdown reaches the target letter (A), participants need to press the target button (last screen, single-button response box). (B) Schematic representation of a task sequence. Up to four-letter series ran, hidden from view (represented as gray letters), at different updating rates and with varying starting letters. Pressing a color-coded monitor button (four-button response box, right) displays the corresponding series for a short period (color-coded frames, black letters). When a series runs down to $A$, the target button (single button response box, right) needs to be pressed. In this example, targets of the blue and yellow letter countdowns were missed (red circles), whereas a target of the red letter countdown was correctly indicated (green circle). countdowns. Monitor buttons and target buttons were assigned to MR-compatible button boxes, with four monitor buttons to be pressed with the fingers of the right hand, and a single target button to be pressed with the left index finger. Countdowns (after pressing the color-matched monitor button) were presented centrally on the screen to reduce eye movements and changes in spatial layout.

To prevent cross-task monitoring (i.e., predicting the state of another countdown) and to match the number of targets between trials with varying numbers of countdowns, each letter series progressed at different rates and covered different series lengths (e.g., $F$ to $A$ at a slow rate or $L$ to $A$ at a faster rate). Updating rates (i.e., the time between two letter changes in a series of a given countdown) were adjusted for the number of countdowns. For example, for the four-countdown trials, the updating rates were 816, 1376, 1536, and $1568 \mathrm{msec}$, respectively. For trials with less than four countdowns, updating rates were randomly selected from the four possible updating rates and adjusted to $3 / 4,1 / 2$, or $1 / 4$ of the updating rate intervals for four countdown trials, for trials with three, two, and one countdowns, respectively. Correspondingly, to warrant similar number of targets per countdown, countdowns started (and restarted after reaching target state) at varying letters, with series lengths of $25,16,11$, and 10 letters for the fastest to slowest updating rates, respectively. Using these parameters, each trial of $60 \mathrm{sec}$ contained 8-10 evenly distributed targets, independently of the number of countdowns, and independently of the randomly selected countdown settings (for trials with less than four countdowns). The assignment between monitor buttons and countdowns (in terms of updating rate/series lengths) was randomized for each trial.

A control task was introduced to control for spatial mapping of stimulus-response associations, visual stimulation, and motor output. Critically, the control task did not require (or enable) time monitoring, as both monitoring and target occurrence was random to the participant. Similar to the experimental task, the control task was presented in trials consisting of one to four random letter series (here referred to as control countdowns). Control countdowns contained random letters (A-Z) that were presented automatically for $1 \mathrm{sec}$ (comparable to actively monitored countdown series during the experimental task). Participants were asked to press the corresponding monitor button upon presentation of a control countdown series and to press the target button when a letter $A$ was shown. The timing of control countdown appearances was based on the active monitoring behavior (experimental task) of a pilot participant. Therefore, the control task provides condition-matched control conditions (i.e., in terms of number of simultaneous countdowns and monitor buttons per condition), visual input, and motor output (both monitor and target button presses, reflecting timing and complexity/number of response options). 
The experiment consisted of five runs, including three runs of the experimental task, intermitted by two runs of the control task (exp-con-exp-con-exp). Experimental task runs consisted of two blocks, and each block contained four trials, one per condition (one to four countdowns). Control task runs consisted of one block with four trials each, one per condition (one to four control countdowns). Trial order within each block was randomized. Each trial lasted $60 \mathrm{sec}$, during which 8-10 targets appeared. A 20-sec break was given between two successive trials/blocks during which a cross-hair was displayed centrally on the screen. After each run (experimental or control task), the scanner was stopped, and a variable break was given to the participant. Participants remained inside the scanner between runs. Participants were informed verbally and visually about the upcoming task (experimental or control), and the experiment continued when participants indicated they were ready to do so.

The main behavioral outcome measure was "monitoring accuracy," calculated as the ratio of successful attempts to indicate the occurrence of target items, relative to all target occurrences. The temporal discrepancy between target occurrence and response was taken as "temporal (in)accuracy." This included early responses (i.e., before the target occurred) as well as late responses (response after target occurred). For further analyses, only absolute values (i.e., temporal offset) were used. Overall monitoring accuracy was calculated over all countdowns within a condition. Moreover, monitoring accuracy was calculated at a countdown level, that is - for each trial-the accuracy on every single countdown. This "countdown-specific monitoring accuracy" was sorted by performance into best, second best, third best, and fourth best countdown (as far as applicable for conditions with less than four countdowns), such that performance of, for example, the best countdowns could be compared between conditions (i.e., depending on the number of additionally monitored countdowns). Only the first target button press was recorded for each countdown series (i.e., between two targets), and data from additional target button presses to the same countdown/target were not considered further. To allow for early and late responses, target button presses that occurred shortly after a target appearance (up to 20\% of the target interval) were assigned to the previous target (i.e., late responses), and the remaining target button presses were assigned to the upcoming target (i.e., early responses). Target button presses that occurred more than $3 \mathrm{sec}$ from the target were removed from the behavioral data analysis because these responses could not reliably be assigned to a specific target or counter $(<1 \%)$. Therefore, only target button presses close to a target occurrence were analyzed. The interquartile range (IQR; Quartile 3-Quartile 1) rule of IQR × 1.5 (Tukey, 1977) was used for detecting the presence of outliers in target button presses. Accordingly, target button presses with discrepancies that were below Quartile $1-1.5$ IQR or above Quartile $3+1.5$ IQR (Tukey, 1977) were removed as outliers (5.22\%). A secondary behavioral outcome measure was "monitoring behavior," specifically the distribution of monitoring actions over time.

\section{Spatial Ability and Working Memory}

Spatial processing and working memory skills were tested in all participants within the large-scale behavioral study reported elsewhere (Kubik et al., 2018, 2019). From these data, measures of spatial ability and working memory performance were used in the current study.

"Spatial ability" was measured with the redrawn version of the mental rotation test (MRT, Version A; Peters et al., 1995; Vandenberg \& Kuse, 1978). This pen-andpaper task contained 12 items. Each item was made up of a target cube construction and four probes. Two of the probes matched the target cube construction, however, rotated around multiple axes relative to the target cube construction; two other probes did not match the target cube construction. The matching cube constructions had to be identified by the participants. It is assumed that participants mentally rotate the figures and try to match them to each other. Participants had 3 min to solve as many items as possible. For each participant, we counted the number of correctly answered items within the 3-min period (with "correct" referring to having identified both matching cube constructions and none of the nonmatching probes).

"Working memory" was measured using verbal and spatial binding tasks (Wilhelm, Hildebrandt, \& Oberauer, 2013). The verbal (letter-color) binding task consisted of 15 trials, in which a short list of letter-color pairs was sequentially presented for $1 \mathrm{sec}$ each (ISI = $3 \mathrm{sec}$ ). Similarly, the spatial (letter-location) binding task consisted of 14 trials, during which a list of letters was presented sequentially for $1.5 \mathrm{sec}$ (ISI $=0.5 \mathrm{sec}$ ) at specific locations within a $3 \times 3$ grid. Load levels ranged from two to six pairs. After each trial, participants were asked to remember the letter-color and letter-location pairs in a cued recall test. In half of the test trials, participants were probed with the letters and had to select/indicate the corresponding color/location; for the other half, participants were probed with the color/location and had to select the corresponding letter (for more details, see Kubik et al., 2018, 2019). The average performance (proportion correct responses) over both tasks was used as measure for participants' ability of working memory binding.

\section{Behavioral Data Analysis}

Behavioral data obtained during the multitasking (countdown) task was analyzed in terms of performance (monitoring accuracy, countdown-specific monitoring accuracy, temporal accuracy) and monitoring behavior. Performance was measured by overall target detection (monitoring accuracy; in line with previous studies Kubik et al., 2018, 2019; Todorov et al., 2014; Mäntylä, 2013) and temporal 
accuracy, a more sensitive measure (see Countdown Task section). Monitoring accuracy and temporal accuracy were analyzed using one-way ANOVAs, with Condition as a within-subject factor, followed by pairwise comparisons between conditions where applicable. Furthermore, countdown-specific monitoring accuracy (see Countdown Task section) was compared between the single best countdowns of each condition (as well as additional counters) between conditions. To test and demonstrate possible relations to spatial processing (as in a spatial offloading mechanism), monitoring accuracy was correlated with participants' spatial abilities (mental rotation task), both for overall performance and for performance on the best counters for each condition. Moreover, following previous studies showing that spatial abilities (MRT) explain multitasking performance (monitoring accuracy) beyond working memory skills and executive functioning (Kubik et al., 2018, 2019), monitoring accuracy was correlated with mental rotation abilities after correction for working memory abilities. Therefore, partial correlations were computed between monitoring accuracy, spatial ability, and working memory.

"Monitoring behavior" was analyzed in terms of monitoring actions per condition, overall (i.e., number of monitoring actions per trial), and over time. One purpose of analyzing monitoring behavior was to verify and demonstrate that participants proactively plan their monitoring behavior. Moreover, by comparing monitoring behavior between conditions, we aimed to demonstrate that demands on time monitoring increase with an increasing number of monitored countdown series. For overall monitoring behavior, monitoring actions were counted for each condition and compared using one-way ANOVAs, followed by post hoc comparisons where applicable. Furthermore, monitoring behavior was analyzed in a time-resolved manner: Monitoring actions were timelocked to target onsets on a countdown-series-specific basis (e.g., timing of monitoring the red countdown relative to the target occurrence of the red countdown) and split in bins of 500-msec duration. Only the final $6 \mathrm{sec}$ (12 bins) before target onset were considered during this analysis, such that all conditions covered the analyzed period (based on target-to-target intervals). Time bins were categorized into "early" bins (6-3 sec before target onset) and "late" bins (1.5-0 sec before target onset). Time-resolved monitoring behavior was analyzed in a two-way ANOVA with factors Time (early, late) and Condition (number of countdowns). A significant Time $\times$ Condition interaction was followed up by time-specific one-way ANOVAs (testing for effects of condition within each epoch; followed by pairwise post hoc comparisons between conditions where applicable). Ratios between early and late monitoring behavior were calculated by dividing the (average) number of monitoring actions during early time bins by the (average) number of monitoring actions during the late time bins for each condition. Ratios were compared between conditions using repeated-measures ANOVA.

\section{MR Data Acquisition and Preprocessing}

A 3T MRI scanner (GE Discovery MR 750; General Electric) with an eight-channel head coil was used for acquiring functional and structural MR images. Anatomical images were acquired using a T1-weighted sequence (repetition time $[\mathrm{TR}] /$ echo time $=6400 / 2800 \mathrm{msec}$, voxel size: $1 \times 1 \times 1 \mathrm{~mm}$ ). BOLD signal was recorded with a whole-brain T2*-weighted EPI sequence (TR/ $\mathrm{TE}=2000 / 30 \mathrm{msec}$; voxel size: $3 \times 3 \times 3 \mathrm{~mm}$, gap size: $0.5 \mathrm{~mm}$ ). The task was divided into three experimental runs of $11.2 \mathrm{~min}$ each and two control runs of 5.7 min each during which 336 and 172 volumes were recorded, respectively. Additional diffusion tensor imaging and resting-state fMRI scans were acquired after the task fMRI scans. These data are not part of the current report.

Images were preprocessed using SPM12 (Wellcome Centre for Human Neuroimaging, University College London, United Kingdom) and ICA-AROMA (Pruim et al., 2015) as part of FSL v5.0 (FMRIB's Software Library). SPM-based preprocessing included spatial realignment, slice time correction, and coregistration of the functional images to the structural image of each participant. All brains were normalized to the Montreal Neurological Institute (MNI) template using the unified segmentation procedure and resampled to $2 \times 2 \times 2 \mathrm{~mm}$ voxel size. Functional images were smoothed using a 6-mm FWHM Gaussian kernel. Next, AROMA was used to remove movement-related artifacts. BOLD signals from white matter, corticospinal fluid, and out of brain areas, obtained from the segmentation in SPM, were extracted and removed from the fMRI time using linear regression, and the data were filtered using a 128-sec highpass filter.

\section{fMRI Data Analysis}

For single-subject analyses, separate general linear models were constructed for each run, modeling trials using boxcar functions, with separate regressors for each condition (i.e., number of countdowns), with a length corresponding to the block length. The purpose of these regressors was to capture underlying state activation during monitoring of one, two, three, or four overlapping task timelines. Additional regressors were constructed to model button presses and visual stimulation. Using FEAT (part of FSL), we first estimated statistical parametric maps corresponding to each condition within each run. Next, in a second-level fixed-effect analysis, we calculated the contrast of BOLD signal during the experimental task and the control task, separately for each condition/ number of countdowns. This resulted in four whole-brain maps per participants, corresponding to the BOLD signal difference between experimental and control task for one-, two-, three-, and four-letter series (i.e., exp1 > con $1, \exp 2>\operatorname{con} 2, \exp 3>\operatorname{con} 3, \exp 4>\operatorname{con} 4$; in the 
remainder referred to as "conditions"), which were used for group-level random effect analyses.

We used complementary approaches for group-level random effect analyses of the functional imaging data. In a "contrast-based approach," we analyzed the activation corresponding to each of the experimental conditions (i.e., monitoring one to four deadlines), compared with the corresponding control conditions, and then directly compared activation maps corresponding to different conditions/numbers of parallel countdowns. In a complementary model-based approach, we identified brain regions that followed linear and step function increases with the number of parallel countdown series and compared the model fits. These analyses are described in detail below.

\section{Part I: Contrast-based Analyses}

We obtained neural activation maps for each experimental condition of the multitask paradigm, corrected for activation during the corresponding control task condition. Specifically, for each condition (i.e., number of parallel countdowns, 1-4), we compared the whole-brain activation maps between the experimental and the control task in a group analysis, treating participants as random effects using nonparametric paired $t$ tests in PALM (Permutation Analysis of Linear Models; Winkler, Ridgway, Webster, Smith, \& Nichols, 2014). The resulting condition-specific activation maps were compared between the different conditions (i.e., number of component tasks, corrected for condition-specific control task activation) using nonparametric $F$ and $t$ tests. First, we performed a one-way within-subject (repeated-measures) ANOVA ( $F$ test) with four levels (reflecting the four conditions). The $F$ test was followed up with condition-specific comparisons (nonparametric paired $t$ tests) using the significant clusters obtained from the $F$ test as mask. Second, we performed whole-brain, condition-specific comparisons. For this comparison, one-tailed one-sample $t$ tests of the difference maps (i.e., 2v1, 3v1, 4v1, 3v2, 4v2, 4v3) were used to identify increased brain activation as a function of increased numbers of parallel monitored countdowns. All inferential statistics were performed using permutation testing (sign flipping) with 1000 random permutations, combined with gamma acceleration to improve $p$ value estimates. Significant clusters were identified using a cluster-defining threshold of $Z>3.1$ (corresponding to $p_{\text {(unc) }}<.001$ ) and cluster-corrected threshold of $p_{(\mathrm{FWE})}<.05$ (Worsley, 2001).

\section{Part II: Model-based Analyses}

For the model-based approach, we identified brain regions that showed activation patterns that corresponded to three proposed theoretical models (Figure 2). Models followed a priori predictions how activation depends on the number of counters in different parts of the brain, following different versions of the spatial offloading hypothesis. Specifically, a linear increase model (Figure 2A), where activity increases linearly with the number of monitored countdowns, a multitask (3+) step model (Figure 2C), with high activation levels for three and more tasks, relative to one and two tasks, and finally, a dual-task step model (Figure 2B), with a high activation level for two and more monitored series, relative to one series. Multitask (or dualtask)-specific spatiotemporal processes should follow a multitask (or dual-task) step model activation pattern, whereas general spatiotemporal processes should follow the linear increase model. Importantly, given that these models are implicitly tested against a null model (i.e., no systematic activation differences depending on the number of monitored countdowns), for spatiotemporal offloading mechanisms to be supported, a model needs to explain activation differences in cortical regions related to spatial processing. The pattern of this activation (e.g., linear vs. multitask step increase) will provide information about the specificity (with respect to dual task or multitask) of such a spatiotemporal offloading process.

For each participant, we used FEAT to obtain modelspecific activation maps from the [experimental-control task] contrast images for each number of component tasks (see Part I: Contrast-based Analyses section). Group average model activation maps were obtained using PALM, using 1000 permutations, gamma acceleration (Winkler,
Figure 2. Models used to identify brain regions following specific activation patterns depending on the number of parallel tasks. Predicted BOLD signal intensity depending on the number of parallel tasks for different tested models, that is, linear increase model (A), dualtask step model (B; brain regions recruited for dual-task+ situations), multitask (3+) step model (C; brain regions recruited for multitasking situations with three and more tasks, but not dual tasking).

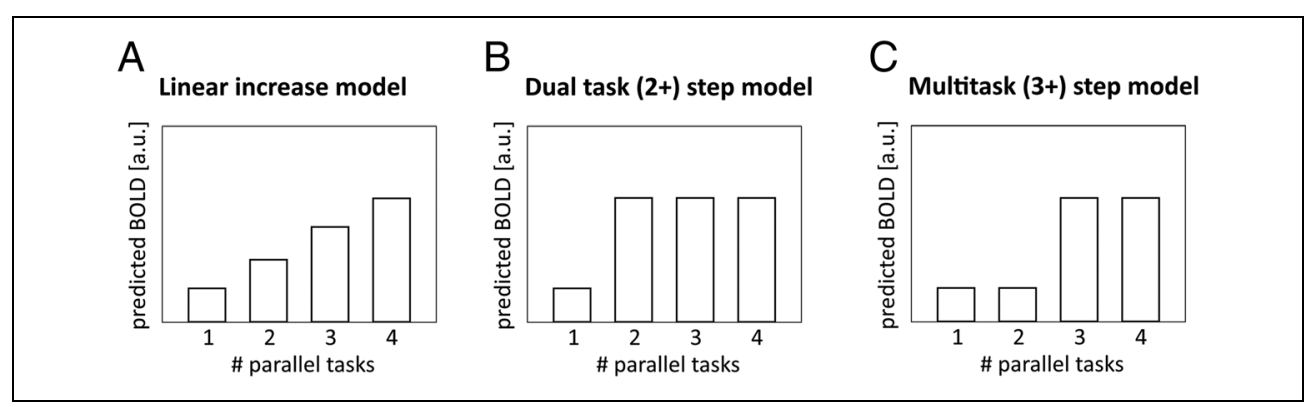


Ridgway, Douaud, Nichols, \& Smith, 2016), and wholebrain masking. Moreover, we obtained differences between model activation maps by pairwise comparison of model-specific activation maps, using PALM (1000 permutations, gamma acceleration, whole-brain masking). We used a cluster-defining threshold of $Z>3.1$ (corresponding to $\left.p_{\text {(unc) }}<.001\right)$ and cluster-corrected threshold of $p_{\text {(FWE) }}<.05$ (Worsley, 2001) to identify significant clusters. Only positive relationships (i.e., activation increases following the models) were considered. For visualization purposes, we extracted parameter estimates from the identified affected regions (i.e., significant clusters in any of the model-based analyses) for each condition-specific neural activation map (i.e., number of countdowns). Mean parameter estimates were extracted from ROIs centered on the peak coordinates of affected regions (sphereshaped, 5-mm radius).

\section{ROI Analysis: Hippocampus}

In addition to whole-brain analyses, we analyzed activation in two ROIs, the left and right hippocampus. ROIs were defined based on a probabilistic atlas (HarvardOxford subcortical structural atlas, part of FSL). ROIs for the left and right hippocampus were based on maximum probability for the left and right hippocampus, with a lower limit requirement of 50\% probability. Parameter estimates of the experimental-control task contrasts were extracted for both ROIs. Parameter estimates were compared between conditions using one-way ANOVAs and fitted to a set of predefined models (see Figure 2). Model fitting was performed using the fitlm function in MATLAB to obtain the Akaike information criterion (AIC; Akaike, 1974) as well as $R^{2}$ values for each model/ROI.

\section{Brain-Behavior Correlations}

To investigate whether interindividual differences in behavioral performance during multitasking can be directly related to interindividual differences in brain function, we correlated measures of behavioral performance with measures of brain activation in the set of affected regions. Specifically, we created ROIs for each affected region (based on any of the model-based analyses; see Modelbased Analyses section), using sphere-shaped ROIs (5-mm radius) centered on the peak coordinates of the affected regions. Parameter estimates of the fourcountdown condition (i.e., multitask-specific activation), as well as the difference between four- and two-countdown conditions (i.e., activation during multitasking corrected for dual tasking), were correlated with corresponding measures of behavioral performance (i.e., monitoring accuracy during multitasking and monitoring accuracy during multitasking divided by monitoring accuracy during dual-tasking condition, respectively) and measures of spatial ability (MRT scores) using Spearman's rank correlations.

\section{RESULTS}

\section{Behavioral Results}

"Monitoring accuracy" (proportion correctly identified target letters) was not significantly affected by the number of monitored countdown series, $F(3,69)=1.17, p=$ $.329, \eta^{2}=.003$. Specifically, participants correctly identified a high number of countdown targets independent of the number of monitored countdown series (one countdown: $0.83 \pm 0.21$ (mean $\pm S D$ ); two countdowns: $0.83 \pm$ 0.19 ; three countdowns: $0.81 \pm 0.19$; four countdowns: $0.81 \pm 0.18$; Figure $3 \mathrm{~A}$ ). However, an analysis of countdownspecific monitoring accuracy (i.e., monitoring accuracy for individual countdown series within each trial) revealed that performance of the best countdown series increased with the number of monitored countdown series (Figure 3B). The same pattern was observed for the second and third best countdown series of the dual- and multitasking conditions. Whereas overall monitoring accuracy did not show significant correlations with mental rotation scores (all $p s>.10$ ), accuracy for the best countdown in each trial did correlate significantly with MRT scores for the multitasking conditions (three countdowns: $r=.459, p=.032$; four countdowns: $r=.511, p=.015$ ), however, not significantly with the single-task condition
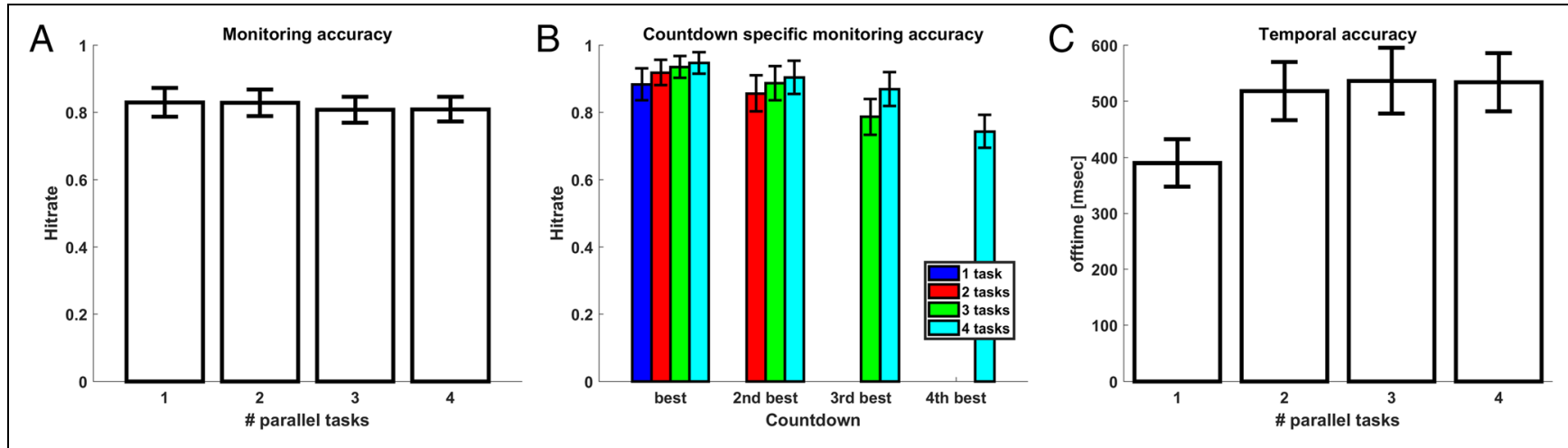

Figure 3. Behavioral performance. (A) Monitoring accuracy, (B) countdown-specific (detailed) monitoring accuracy, and (C) temporal accuracy. Error bars indicate SEM. 


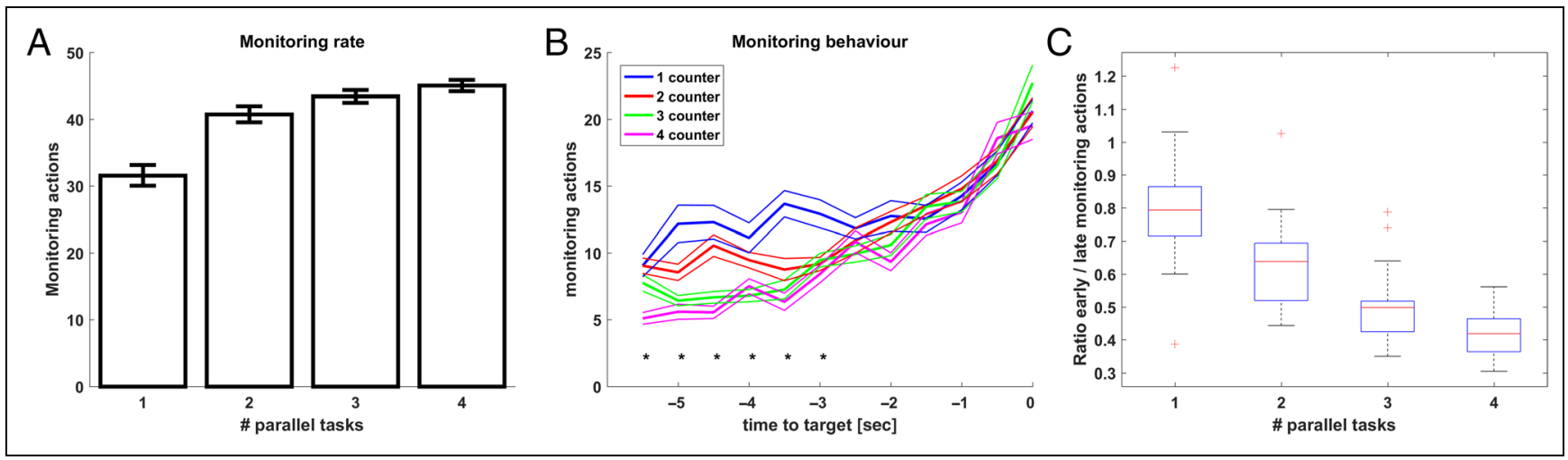

Figure 4. Monitoring behavior. (A) Monitoring actions by condition/number of monitored counters. (B) Monitoring behavior relative to target occurrence, depending on number of monitored countdowns. Thick lines represent monitoring actions binned in 500-msec windows, relative to target occurrence (up to $6 \mathrm{sec}$ before target occurrence). Thin lines represent standard errors of the mean. Asterisks indicate time bins with significant differences between conditions ( $F$ test, one-way ANOVA; $p<.01$ ). (C) Ratio between early (Bin $1-6$ [5.5-3 sec before target occurrence]) and late (Bin 10-12, 1.5-0 sec before target occurrence) time bins, depending on number of monitored countdowns. Error bars indicate SEM.

$(r=.373, p=.087)$ and dual-task condition $(r=.420, p=$ .052). Partial correlations between MRT and monitoring accuracy, controlling for individual working memory skills, were significant for the best countdown in the four-countdown condition $(r=.467, p=.033)$. Partial correlations for best countdowns of other conditions were not significant (three countdowns: $r=.429, p=$ .053; two countdowns: $r=.405, p=.069$; one countdown: $r=.366, p=.103$ ). Performance for added countdowns (i.e., second best, third best, fourth best countdown series) did not correlate with MRT scores (all $p s>.10$ ), and none of the partial correlations for second, third and fourth best countdowns were significant (all $p s>.10$ ).

"Temporal accuracy" was significantly affected by the number of monitored countdown series, $F(3,69)=26.08$, $p<.001, \eta^{2}=.056$. Specifically, the temporal accuracy at which targets were marked decreased with a higher number of monitored countdowns (Figure 3C). In detail, temporal accuracy was higher for one countdown (390 $\pm 207 \mathrm{msec})$ compared with two countdowns (518 $\pm 256 \mathrm{msec}), t(23)=$ $6.00, p<.001$; three countdowns (536 $\pm 288 \mathrm{msec}), t(23)=$ $5.48, p<.001$; and four countdowns (534 $\pm 256 \mathrm{msec})$, $t(23)=7.81, p<.001$. There were no significant differences between temporal accuracy for two, three, and four countdowns (all ps >.10; all Bonferroni corrected).

"Monitoring behavior" was significantly affected by the number of monitored countdown series. Generally, participants performed more monitoring actions with increasing number of monitored countdown series, $F(3$, 69) $=116.19, p<.001, \eta^{2}=.465$. Specifically, monitoring actions increased with each condition (i.e., from two to one countdown: $t(23)=13.63, p<.001$; three to two countdowns: $t(23)=4.49, p=.001$; four to three countdowns: $t(23)=5.16, p<.001)$. Average number of monitoring actions per trial was $31.60 \pm 7.6$ during the one-countdown condition, $40.76 \pm 5.79$ during the two-countdown condition, $43.43 \pm 4.54$ during the three-countdown condition, and $45.04 \pm 4.24$ during the four-countdown condition
(Figure 4A). A temporal analysis of monitoring behavior (Figure 4B) suggested that monitoring of a specific countdown series (relative to its target occurrences) was similar close to target occurrence but differed in the time before target occurrence. This pattern was confirmed by a significant interaction of monitoring actions between time (early vs. late within a countdown series) and condition (number of monitored countdown series), $F(3,69)=44.64, p<$ .001. The interaction was driven by a significant difference between conditions during the early epoch (Time Bins $1-6), F(3,69)=48.32, p<.001$, and the absence of a significant difference during the late epoch (Time Bins 10-12), $F(3,69)=0.39, p=.761$. During the early epoch, pairwise comparisons of monitoring behavior indicated significant decreases in monitoring activity with each added countdown series (two vs. One countdown: $t(23)=4.36, p=$ .001 ; three vs. two countdowns: $t(23)=7.22, p<.001$; four vs. three countdowns: $t(23)=3.89, p=.002$; all Bonferroni corrected).

For each participant and condition, we calculated the ratio between early and late monitoring actions to measure the impact of increased demands on time monitoring on monitoring behavior. The ratio of early monitoring actions relative to late monitoring actions decreased with the number of monitored counters (Figure 4C), meaning that participants perform more monitoring actions when they monitored fewer countdowns, relative to monitoring actions that occurred in close temporal proximity to the target. The decrease can be described by linear regression $(\beta=-.758, F(1,23)=127.817, p<.001$, $\eta^{2}=.847$; quadratic: $\left.F(1,23)=9.342, p=.006, \eta^{2}=.289\right)$.

\section{Brain Imaging Results}

\section{Part I: Condition-specific Brain Activation}

First, to identify the neural networks involved in monitoring of task time and deadlines during single-, dual-, and 
Figure 5. Condition-specific activation maps. Significant clusters (pFWE <. 05; cdt: $z=$ 3.1) from contrasts between experimental and control task for each condition (i.e., number of countdowns) are shown. (A) Single-task (one countdown), (B) dual-task (two countdowns), (C) multitask (three countdowns), and (D) multitask (four countdowns). The reverse contrasts [controlexperimental] did not result in any significant clusters.

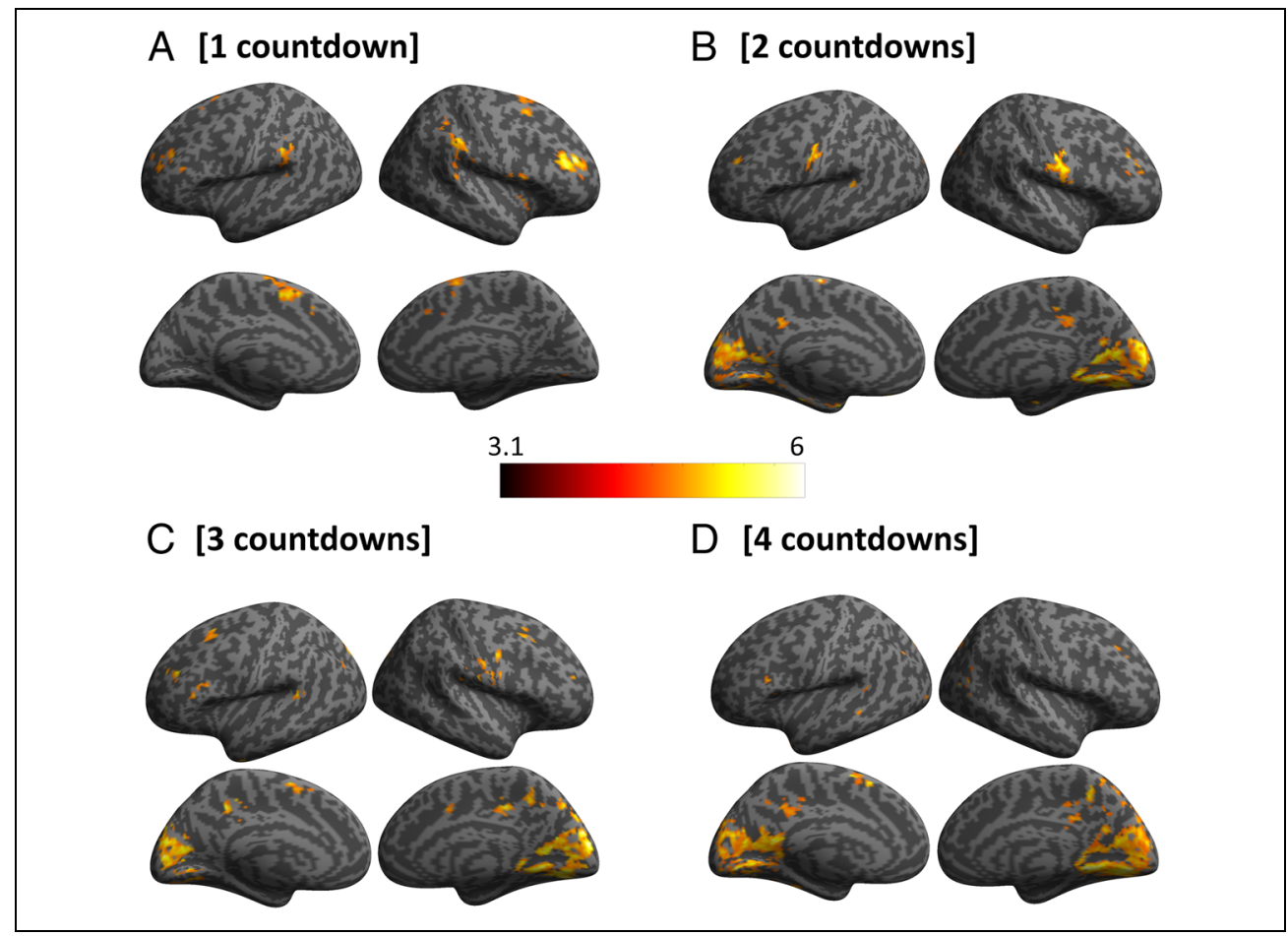

Table 1. Condition-specific Comparisons

\begin{tabular}{|c|c|c|c|c|c|c|}
\hline Contrast/Region & Voxels & $Z \operatorname{Max}$ & $X(m m)$ & $Y(m m)$ & $Z(m m)$ & Cluster $p$ \\
\hline \multicolumn{7}{|l|}{$4>1$} \\
\hline $\mathrm{L}$ anterior IPS/IPL & 96 & 4.932 & -34 & -62 & 35 & .018 \\
\hline $\mathrm{R}$ posterior cingulate gyrus & 78 & 4.279 & 2 & -36 & 32 & .019 \\
\hline $\mathrm{R}$ medial frontal cortex & 64 & 5.299 & 4 & 52 & -20 & .021 \\
\hline $\mathrm{R}$ middle temporal gyrus & 60 & 5.031 & 54 & -4 & -20 & .021 \\
\hline $\mathrm{L} / \mathrm{R}$ precuneus & 60 & 4.310 & -2 & -54 & 38 & .022 \\
\hline L occipital pole (V2) & 58 & 4.624 & -26 & -100 & -10 & .022 \\
\hline L occipital pole (V2) & 50 & 4.519 & -10 & -88 & 32 & .024 \\
\hline $\mathrm{R}$ anterior IPS/IPL & 47 & 4.147 & 36 & -64 & 44 & .024 \\
\hline $\mathrm{R}$ posterior cingulate gyrus & 46 & 4.625 & 4 & -50 & 8 & .024 \\
\hline R hippocampus & 35 & 4.857 & 26 & -26 & -18 & .028 \\
\hline L middle temporal gyrus & 32 & 4.605 & -62 & -42 & 4 & .030 \\
\hline L IPL & 32 & 5.080 & -52 & -74 & 26 & .030 \\
\hline L posterior cingulate gyrus & 29 & 4.556 & -6 & -44 & 0 & .033 \\
\hline R occipital pole (V2) & 28 & 5.248 & 18 & -88 & -10 & .031 \\
\hline $\mathrm{R}$ middle temporal gyrus & 25 & 4.083 & 64 & -4 & -24 & .042 \\
\hline \multicolumn{7}{|l|}{$3>1$} \\
\hline $\mathrm{L} / \mathrm{R}$ precuneus & 80 & 4.758 & 2 & -54 & 34 & .050 \\
\hline
\end{tabular}

Clusters for comparisons with significant activation differences are listed (cluster forming threshold: $z=3.1$; measure: cluster mass). FWE-corrected cluster-level $p$ values are shown based on permutation testing (PALM; see Methods section). $\mathrm{L}=$ left; $\mathrm{R}=$ right. 


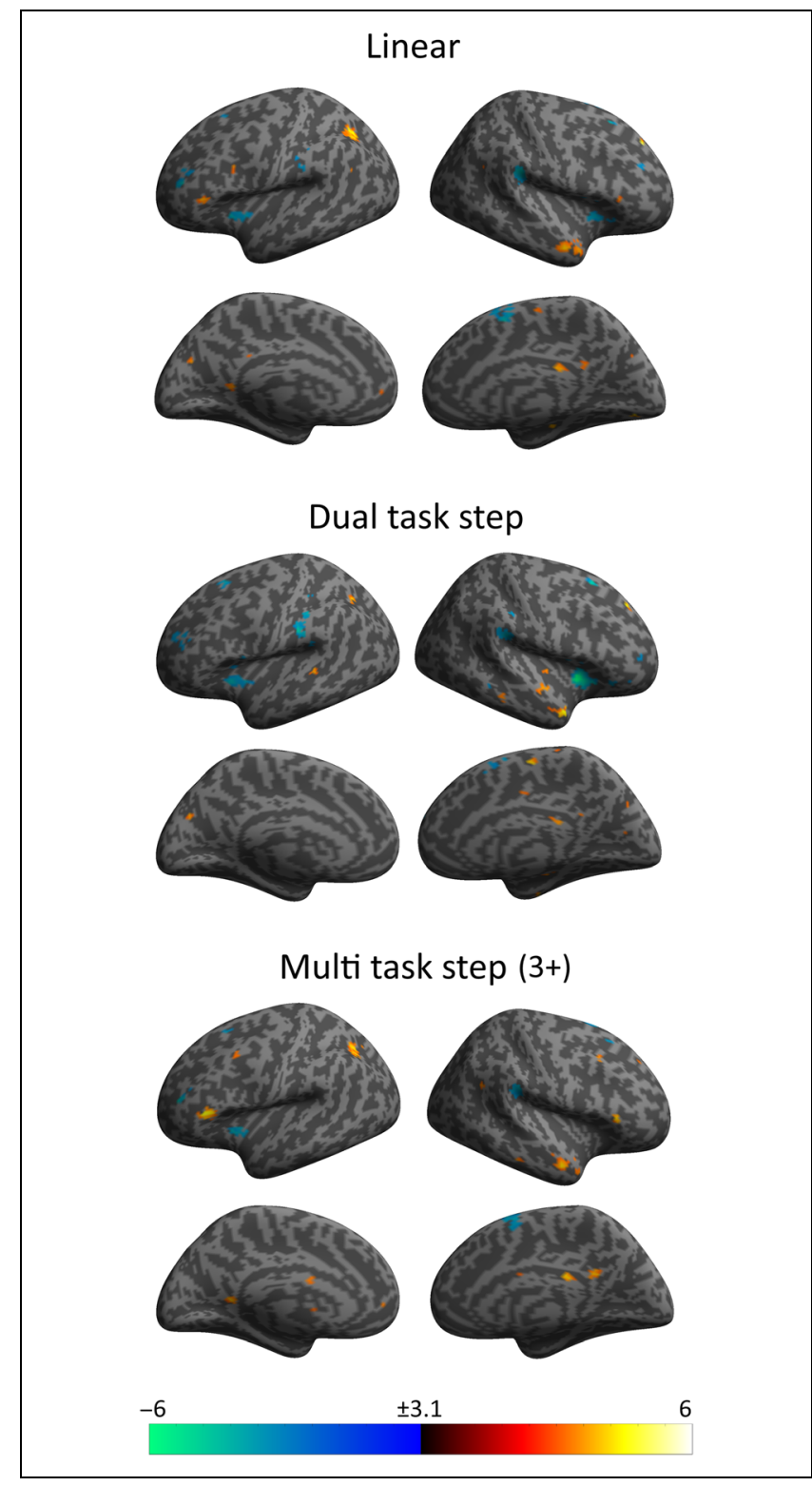

Figure 6. Model-specific activation maps. Activation following positive (red-yellow) and negative (bluegreen) models. Top: linear model. Middle: dual task step model. Bottom: Multitask $(3+)$ step model. Displayed voxel wise $t$ values $>3.1$ (uncorrected). See Table 2 for cluster-level statistics and details.

multitasking, we obtained neural activation maps for each condition of the multitasking paradigm, corrected for activation during the corresponding control task condition (see Methods-Part I: Contrast-based Analyses section). For the single-countdown condition, the experimental task, relative to the control task, activated middle cingulate cortex and the SMA, bilateral middle frontal gyrus, bilateral superior temporal gyrus and pole, the right inferior parietal cortex, the left superior frontal gyrus, and parts of the cerebellum (Figure 5A). With two monitored countdowns, the experimental task, relative to the control task, activated extensively the visual cortex bilaterally, as well as the left and right postcentral gyri, the middle and superior frontal gyri, the SMA and fusiform gyri, as well as the left superior temporal gyrus and the left parahippocampal gyrus (Figure 5B). Similar results were obtained for the condition with three countdowns, activating extensively the visual cortex, the middle and inferior frontal gyri, the SMA and midcingulate area, the pre- and postcentral cortex bilaterally, as well as the left superior and inferior temporal gyri (Figure 5C). The four-countdown condition activated extensively the visual cortex, the fusiform gyrus, the SMA, the middle temporal gyri and poles bilaterally, and the middle and frontal gyri (Figure 5D). None of the reverse contrasts ([control-experimental] for each of the conditions) resulted in significant clusters.

\section{Condition-specific Comparisons}

Next, activation was compared between conditions. We observed a significant cluster in the precuneus in a one-way ANOVA including all four conditions testing for overall differences between the four conditions (MNI: $\left.[2-5434] ; F=543.24, p_{(\mathrm{FWE})}=.04\right)$. Follow-up comparisons using the significant cluster as mask revealed no step-wise differences (i.e., $2>1,3>2,4>3$; all $p$ s $>$ .05 ; however, all $p$ s $<.10$; all FWE-corrected). However, there were significant differences in comparisons between three- and one-countdown conditions $(p=.003)$, between four- and one-countdown conditions $(p<.001)$, and between four- and two-countdown conditions ( $p=$ .003). Similarly, whole-brain paired $t$ tests revealed no significant clusters for step-wise comparisons (i.e., $2>1,3>$ $2,4>3$ ). However, a significant cluster in the precuneus was observed when comparing multitask conditions with three and four parallel countdown series with the single countdown condition (Table 1). When comparing the four-countdown multitask condition with the single countdown condition, additional clusters were observed in bilateral inferior parietal cortex/intraparietal sulcus, bilateral middle temporal gyrus, medial frontal cortex, bilateral occipital cortex, posterior cingulate, and the right parahippocampal gyrus (Table 1). In the whole-brain comparisons, no differences were observed between the multitask conditions (three and four countdowns) and the twocountdown condition.

\section{Part II: Model-specific Activation Maps}

In a complementary approach, we identified brain regions in accordance to model-based activation patterns. These results are presented in Figure 6 and Table 2. The linear increase model (Figure 2A) was associated with activation in the left inferior parietal lobe (IPL; extending into the angular gyrus; $p=.013$ ) and nonsignificant clusters in the bilateral precuneus $(p=.056)$ and the right middle temporal gyrus $(p=.063)$. Similarly, the dual-task step model $(2+$; Figure $2 \mathrm{~B})$ predicted significant clusters of increasing activation in the left IPL $(p=.039)$ and 
Table 2. Model-specific Activation Patterns

\begin{tabular}{|c|c|c|c|c|c|c|}
\hline Region & Voxels & $Z \operatorname{Max}$ & $X(m m)$ & $Y(m m)$ & $Z(\mathrm{~mm})$ & Cluster $p$ \\
\hline \multicolumn{7}{|l|}{ A. Linear Increase Model } \\
\hline$L$ inferior parietal & 308 & 6.81 & -34 & -62 & 36 & .013 \\
\hline$L / R$ precuneus & 132 & 4.88 & -2 & -54 & 38 & .056 \\
\hline$R$ middle temporal & 118 & 5.22 & 54 & -6 & -20 & .063 \\
\hline $\mathrm{R}$ middle cingulum & 75 & 4.26 & 2 & -36 & 32 & .101 \\
\hline $\mathrm{R}$ inferior medial frontal lobe & 60 & 5.21 & 4 & 52 & -20 & .117 \\
\hline $\mathrm{R}$ angular gyrus & 54 & 4.47 & 36 & -64 & 44 & .131 \\
\hline $\mathrm{L}$ angular gyrus & 50 & 6.91 & -52 & -74 & 26 & .133 \\
\hline cerebellum vermis & 26 & 4.27 & 2 & -52 & 10 & .226 \\
\hline $\mathrm{R}$ lingual & 14 & 5.46 & 18 & -88 & -8 & .441 \\
\hline \multicolumn{7}{|l|}{ B. Dual-task Step Model } \\
\hline$L$ inferior parietal & 104 & 5.50 & -32 & -72 & 46 & .039 \\
\hline$L / R$ precuneus & 54 & 4.29 & -2 & -54 & 38 & .060 \\
\hline$L$ inferior medial frontal lobe & 33 & 4.76 & -2 & 46 & -26 & .082 \\
\hline $\mathrm{R}$ middle temporal & 24 & 5.19 & 54 & 2 & -26 & .106 \\
\hline $\mathrm{R}$ posterior cingulum & 24 & 4.15 & 2 & -34 & 30 & .117 \\
\hline \multicolumn{7}{|l|}{ C. Multitask (3+) Step Model } \\
\hline $\mathbf{L} / \mathbf{R}$ precuneus & 257 & 5.08 & 4 & -54 & 34 & .023 \\
\hline$L$ inferior parietal & 148 & 4.79 & -34 & -74 & 44 & .045 \\
\hline$R$ middle temporal & 116 & 5.01 & 52 & -10 & -18 & .057 \\
\hline $\mathrm{R}$ posterior cingulum & 52 & 4.40 & 10 & -36 & 30 & .112 \\
\hline $\mathrm{R}$ middle frontal gyrus & 50 & 4.41 & 36 & 22 & 44 & .114 \\
\hline L inferior frontal gyrus (pars triangularis) & 45 & 5.00 & -54 & 34 & 10 & .118 \\
\hline $\mathrm{L}$ angular gyrus & 39 & 5.89 & -52 & -74 & 26 & .132 \\
\hline L middle frontal & 32 & 4.43 & -22 & 20 & 38 & .156 \\
\hline $\mathrm{R}$ angular gyrus & 22 & 3.97 & 42 & -68 & 48 & .24 \\
\hline
\end{tabular}

Clusters for each model are listed (cluster forming threshold: $z=3.1$; measure: cluster mass). FWE-corrected cluster-level $p$ values are shown, based on permutation testing (PALM, see Methods section). Significant clusters $(p<.05)$ are printed in bold. Non-significant clusters $(p<.10)$ are printed in italics. $\mathrm{L}=$ left; $\mathrm{R}=$ right.

nonsignificant clusters in the left and right precuneus $(p=.060)$ and the left inferior medial frontal lobe $(p=$ .082). The multitask (3+; Figure 2C) step model predicted activation in the precuneus $(p=.023)$ and the left IPL $(p=.045)$, and nonsignificant clusters in the right middle temporal gyrus $(p=.057)$. Generally, there was extensive overlap between the activation maps of all models, given the similarity of the models.

ROIs were generated based on the significant clusters obtained from the model-specific activation maps (Table 2) for illustration purposes (Figure 7) and for calculation of model fits. These ROIs represented the left inferior parietal cortex (center MNI [-34 -68 42]; significant cluster in the linear increase model, dual-task step model, and multitask $(3+)$ step model), and the precuneus (MNI $[0-54$ 36]; significant cluster in multitask (3+) step model). Visual inspection of extracted parameter estimates generally showed increasing activation with increased number of monitored countdowns in each ROI. Model fit for the inferior parietal ROI was highest for the linear increase model (AIC $=11.26$, $R^{2}=.95$ ), followed by the dual-task step model (AIC $=$ $17.86, R^{2}=.72$ ) and the multitask step model (AIC $=$ $19.33, R^{2}=.59$ ). For the precuneus ROI, model fit was highest for the linear increase model (AIC $=6.37, R^{2}=.99$ ), 


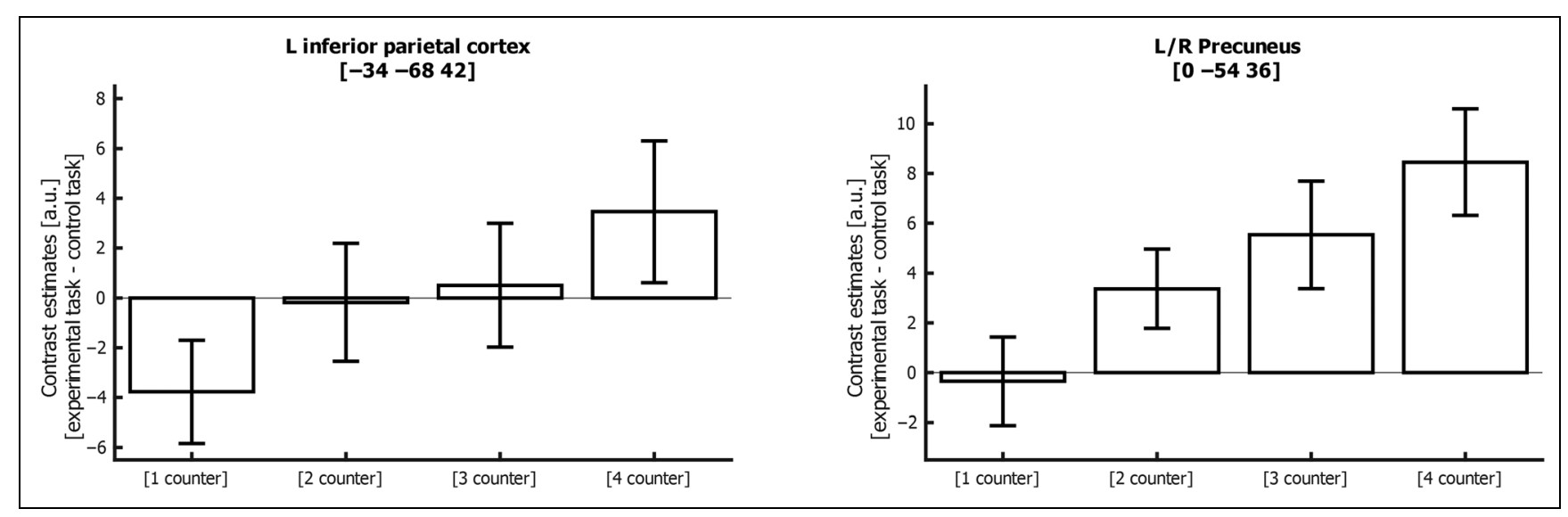

Figure 7. Parameter estimates of selected regions from model activation maps for illustration. Post hoc comparisons of the experimental versus control task conditions (i.e., parameter estimates $<>0$ ) indicate that none of the parameter estimates was significantly smaller than zero (all $p$ s $>.05$, uncorrected), indicating that the control condition did not result in stronger activation than the experimental condition for any of the conditions, within the ROIs. Error bars indicate SEM.

followed by the multitask step model (AIC $=19.45, R^{2}=$ .73) and dual-task step model (AIC $=20.07, R^{2}=.68$ ). No correlations between behavioral measures (multitask performance, mental rotation ability) and brain activation within these ROIs were detected (all $p s>.10$ ).

\section{Model Comparisons}

Whereas the cluster in the precuneus was significant only for the multitask $(3+)$ step model (Table 2), no significant differences between model activation maps of the multitask step model, the dual-task step model, and the linear increase model were observed.

\section{ROI: Hippocampus}

In addition to the whole-brain, model- and contrast-based analyses, we analyzed condition-dependent differences in the hippocampal gyri based on its role on spatial processing (Eichenbaum, 2017). No significant differences between conditions were observed for the left or right hippocampus (one-way ANOVA; all ps > .10; Figure 8). However, for both left and right hippocampus, model fit was best for the dual-task $(2+)$ step models (left: AIC $=$ $4.91, R^{2}=.77$; right: $\mathrm{AIC}=4.72 ; R^{2}=.80$ ), followed by the linear increase models (left: $\mathrm{AIC}=5.57, R^{2}=.73$; right: $\mathrm{AIC}=8.49, R^{2}=.48$ ), and the multitask step models (left: $\mathrm{AIC}=9.43, R^{2}=.30$; right: $\mathrm{AIC}=10.64, R^{2}=.11$ ). No correlations with behavioral performance were observed (all $p s>.10$ ).

\section{DISCUSSION}

The main goal of this study was to identify the neural correlates underlying multiple-task performance. Specifically, we tested a central assumption of the spatial offloading hypothesis, namely whether keeping track of multiple task deadlines relies on spatial processes.

Overall monitoring accuracy was constantly high across the different numbers of countdowns, but detailed analyses suggested that participants adapt their behavior in response to changing task demands. Specifically, when

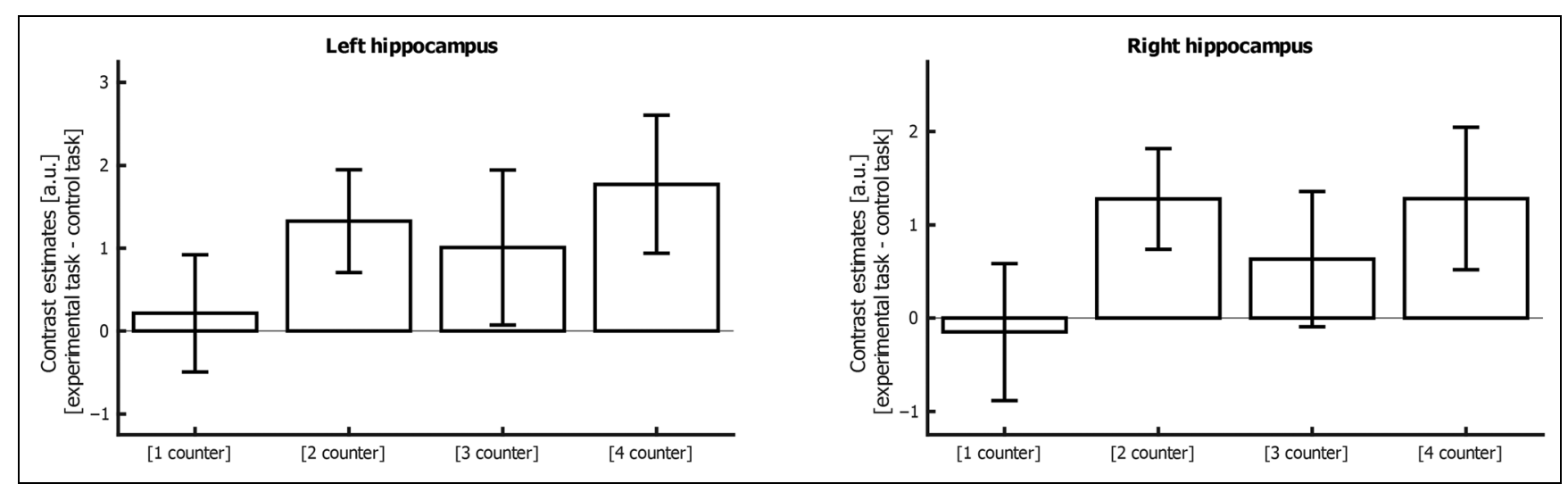

Figure 8. Parameter estimates of the left and right hippocampus ROIs. No significant differences between parameter estimates were observed, however, model-based analyses indicated that the dual-task $(2+)$ step model had the best fit with the data. Error bars indicate SEM. 
the number of countdowns in the task increased, performance for the best countdown in each task condition increased. Performance on additional countdowns (second, third, fourth best) was reduced, resulting in similar overall performance across task conditions (Figure 3B). This pattern may be the result of strategic choices of the participants, in which they increased effort with more countdowns, while at the same time focusing on a subset of component tasks. Such behavioral facilitation from increasing task demands were recently demonstrated within the context of working memory and executive control (Samrani, Marklund, Engström, Broman, \& Persson, 2018). Moreover, and partly in line with previous studies (Kubik et al., 2018, 2019; Todorov et al., 2014; Mäntylä, 2013), performance on this best countdown was predicted by participants' spatial (mental rotation) abilities, even after controlling for working memory (binding) skills. Importantly, this relation became stronger as a function of increased number of monitored countdowns, suggesting that spatial abilities become increasingly important in more complex multitasking situations, which require a higher degree of time monitoring due to multiple overlapping task timelines. An analysis of participants' monitoring behavior further showed that, despite an overall increased monitoring activity, participants reduced their monitoring of each single countdown a relatively long time before the target occurred, whereas monitoring of specific countdowns was not affected by the number of (additionally) monitored countdowns in close temporal proximity to the target (Figure 4). This pattern of monitoring behavior suggests that participants had to rely more on internal representations of the countdowns (rather than monitoring/using external sources of information to retrieve the state of a countdown), when more countdowns had to be monitored. As hypothesized in the introduction, such internal representations may be offloaded onto spatial processes to achieve the same level of performance.

Importantly, the behavioral performance data were accompanied by increased neural activation in the precuneus and IPL, in which the BOLD signal increased as a function of task complexity. Both these regions were more strongly activated when participants monitored and responded to a larger number of parallel tasks. Together, these findings support the idea that multiple task timelines are represented spatially and are in line with the spatial offloading hypothesis by showing that (a) maintaining high behavioral performance is related to individuals' spatial abilities, especially in situations with multiple overlapping task timelines (as in multitask situations) and (b) keeping track of multiple overlapping task timelines involves cortical regions critical for spatial processing (see below).

A secondary goal of this study was to investigate whether processing of task timelines using spatial representations is a general mechanism for time monitoring, or whether such spatial offloading is a mechanism involved in keeping track of task timelines specifically in multitasking situations (Mäntylä, 2013). Here, using a model-based approach, we did not observe significant differences between various models designed to distinguish between predicted neural consequences of these possibilities. In fact, dual- and multitask-specific models, as well as a general (linear increase) model, were all associated with increased activation in a similar set of brain regions. For the identified core regions in the inferior parietal cortex and precuneus, a linear increase was the best fit for the data; however, no specific pattern of brain activation could be exclusively related to a multitaskingspecific mechanism. Therefore, our results do not provide conclusive support for the spatial offloading hypothesis as a mechanism for time monitoring specifically during multitasking. Rather, spatial offloading should be assumed to be a general mechanism for time monitoring across many task situations.

\section{Temporal States and Spatiotemporal Offloading in the Posterior Parietal Cortex and Hippocampus}

There is much evidence that the posterior parietal cortex is important for spatial processing, including sensory and motor control, spatial cognition, and spatial transformations (Sack, 2009; Milner \& Goodale, 2008; Zacks, 2008; Halari et al., 2006; Jordan et al., 2001; Kesner \& Long, 1998; Richter et al., 1997; Cohen et al., 1996; Goodale \& Milner, 1992). However, the role of the posterior parietal cortex is not restricted to spatial processing per se. In fact, the intraparietal lobe is also critical for integrating spatial and temporal information (Assmus et al., 2003), and overlapping areas in IPL have been shown to process spatial and temporal information (Gijssels et al., 2013). Moreover, processes underlying common magnitude systems are thought to be located in the lateral posterior parietal cortex (Sokolowski, Fias, Bosah Ononye, \& Ansari, 2017). Based on these findings and the observation that cortical activation in IPL increases with the number of tasks performed in parallel, IPL may be involved in spatiotemporal offloading. Specifically, temporal information about the states of multiple tasks may be represented in general magnitude systems based on spatial processing areas in the lateral posterior parietal cortex.

Temporal information about single, ongoing tasks may be processed and/or maintained in the precuneus. Indeed, there is evidence for a role of precuneus in temporal processing from studies showing that this region is linked to time estimation over long intervals (Morillon, Kell, \& Giraud, 2009). Specifically, the precuneus, as part of the default mode network, has been associated with time estimation for relatively long event durations exceeding $2 \mathrm{sec}$, whereas shorter intervals are estimated by the motor system (Morillon et al., 2009). Increasing activation in the precuneus with the number of tasks, as observed in the current study, suggests that the 
precuneus estimates the temporal progress of multiple tasks when necessary.

In addition to regions in the posterior parietal cortex, the hippocampus also showed increased activation during dual- and multitask conditions, compared with single-task condition (Figures 3 and 6). In contrast to IPL and precuneus, however, activation in the hippocampi does not increase with additional tasks. The hippocampus is known to be involved in spatial processes, including memory, navigation, and mapping (Eichenbaum, 2017).

Taken together, we suggest that the precuneus, the left IPL, and the hippocampus are core regions for processing of multiple tasks with overlapping timelines. The precuneus, in line with studies relating the precuneus to temporal processing of long ( $>2 \mathrm{sec}$ ) intervals (Morillon et al., 2009), may be involved in temporal processing of multiple task timelines. The IPL may then form relations between different task timelines, using spatial representations and transformations, thus shifting cognitive load of temporal processing onto spatial processes through spatiotemporal offloading (Risko \& Gilbert, 2016; Mäntylä, 2013). The hippocampus, based on the spatiotemporal transformation in IPL, may generate and/or maintain a spatial map of the temporal relations within a multitasking situation and/or may be involved in storage of multiple tasks of such maps over the course of the experiment. The causal contribution of each of these regions remains to be tested experimentally.

\section{Contributions of Medial Frontal, Occipital, and Temporal Cortex}

We also observed increased activation outside spatial processing areas of the posterior parietal cortex during the experimental task conditions compared with the condition-specific control conditions, especially in the medial frontal lobe and the hippocampi (Figure 3 and Figure 6). However, here we did not observe that activation in frontal cortex scaled with the number of task instances (i.e., monitored countdowns). This may be the case because in this study participants did not switch between different task rules and task sets, or stimulus features, as is commonly necessary in dual- and multitask research (von Bastian \& Druey, 2017). Furthermore, we observed increased activation in temporal and occipital regions during the experimental task, compared with the control task (Figure 3). Activation of the temporal cortices may be related to language processing (specifically, processing of letters) and alphabetically structured stimuli (Thesen et al., 2012; Heinzel et al., 2008), considering that temporal areas were activated consistently over all complexity levels, without systematic increases. Whereas the control condition included letters, these did not have to be processed to the same extent. In fact, during the control task, participants only had to recognize the letter $A$ and could ignore any other letter. Finally, stronger activation in visual areas during the experimental task conditions, compared with their respective control conditions, is most likely related to differences in visual attention to the (matched) visual input.

\section{Interpretational Considerations and Limitations}

Posterior parietal regions such as precuneus and IPL have been related to spatial attention and motor control (Lee et al., 2013; Singh-Curry \& Husain, 2009). Even though spatial features were largely controlled for, compared with previous studies on this topic, it can be argued that the different letter series were mapped onto four different fingers. Moreover, in the current task and, in particular, in conditions with higher number of parallel countdowns, this may have increased the demands for spatial motor coordination in the sense that participants had to perform the task with two hands and up to four fingers on one of the hands. However, two aspects argue against such an explanation. First, we introduced condition-specific control tasks, which were similar in terms of motoric complexity in the sense that both hands had to be used, and varying numbers of button presses were executed depending on the control condition. Overall, motor output was similar between the experimental and control tasks. Another important aspect that argues against a motor complexity explanation of posterior parietal activation increases is the lack of activation in other known motor areas, such as premotor and primary motor cortices.

Moreover, it can be argued that the involvement of spatial processes in this task (both in terms of neural activation and correlation with measures of spatial abilities, i.e., mental rotation) were a direct consequence of the task design and the participants' strategies to perform the task. Specifically, one option is that, given the association between (spatially distinct) response buttons and task deadlines/countdowns, task timelines were represented in these spatially distinct locations (e.g., finger locations). To this end, the current study cannot clearly distinguish between a direct neurally implemented spatial offloading mechanism and an alternative embodied offloading strategy. That is, participants may strategically chose to represent the different timelines spatially onto their fingers used for responding. However, we argue that both implementations should be considered forms of a spatial offloading mechanism. Importantly, both mechanisms would be realized through recruitment of cortical spatial processing areas, as observed in this study.

The interpretation in this study, that spatial processing areas are involved in keeping track of multiple timelines, is based upon observation of increased activation in areas commonly known for spatial processing. However, given that participants did not complete a spatial processing task (such as the mental rotation task) during fMRI scanning, we were not able to examine whether spatial tasks and the deadline monitoring task used in the current 
study engage overlapping brain regions. Moreover, the posterior parietal cortex has also been associated with working memory processes, especially working memory updating (Borst \& Anderson, 2013; Wager \& Smith, 2003). It is likely that working memory demands also increase with the number of monitored countdowns (i.e., participants need to keep track of a higher number of letters). Therefore, additional research is required where spatial tasks and working memory tasks are tested in the same participants as time-monitoring tasks using functional neuroimaging techniques.

\section{Conclusion}

This study provides evidence that the precuneus represents timelines of multiple tasks and the IPL is involved in building spatial representations of the temporal relations of these overlapping task timelines. These results are in line with the spatiotemporal offloading hypothesis of multitasking, suggesting that spatial processes are used to resolve temporally demanding tasks when dealing with overlapping task timelines. Therefore, this study enhances our understanding of the neurocognitive mechanisms underlying everyday performance with multiple tasks and overlapping deadlines.

Reprint requests should be sent to Marius Zimmermann, DTU Compute, Technical University of Denmark, Richard Petersens Plads 321, 2800 Kgs. Lyngby, Denmark, or via e-mail: marz@ dtu.dk.

\section{REFERENCES}

Akaike, H. (1974). A new look at the statistical model identification. IEEE Transactions on Automatic Control, 19, 716-723.

Assmus, A., Marshall, J. C., Ritzl, A., Noth, J., Zilles, K., \& Fink, G. R. (2003). Left inferior parietal cortex integrates time and space during collision judgments. Neuroimage, 20 Suppl. 1, S82-S88.

Bonato, M., Zorzi, M., \& Umiltà, C. (2012). When time is space: Evidence for a mental time line. Neuroscience $\varepsilon$ Biobehavioral Reviews, 36, 2257-2273.

Borst, J. P., \& Anderson, J. R. (2013). Using model-based functional MRI to locate working memory updates and declarative memory retrievals in the fronto-parietal network. Proceedings of the National Academy of Sciences, U.S.A., 110, 1628-1633.

Burgess, P. W., Veitch, E., de Lacy Costello, A., \& Shallice, T. (2000). The cognitive and neuroanatomical correlates of multitasking. Neuropsychologia, 38, 848-863.

Cohen, M. S., Kosslyn, S. M., Breiter, H. C., DiGirolamo, G. J., Thompson, W. L., Anderson, A. K., et al. (1996). Changes in cortical activity during mental rotation. A mapping study using functional MRI. Brain: A Journal of Neurology, 119, 89-100.

Collette, F., Olivier, L., Van der Linden, M., Laureys, S., Delfiore, G., Luxen, A., et al. (2005). Involvement of both prefrontal and inferior parietal cortex in dual-task performance. Cognitive Brain Research, 24, 237-251.

Craik, F. I. M., \& Bialystok, E. (2006). Planning and task management in older adults: Cooking breakfast. Memory $\mathcal{E}$ Cognition, 34, 1236-1249.
Dehaene, S., \& Brannon, E. M. (Eds.) (2011). Foreword: Space, time and number in the brain (pp. ix-xii). Cambridge, MA: Academic Press.

Deprez, S., Vandenbulcke, M., Peeters, R., Emsell, L., Amant, F., \& Sunaert, S. (2013). The functional neuroanatomy of multitasking: Combining dual tasking with a short term memory task. Neuropsychologia, 51, 2251-2260.

Eichenbaum, H. (2017). The role of the hippocampus in navigation is memory. Journal of Neurophysiology, 117, 1785-1796.

Gijssels, T., Bottini, R., Rueschemeyer, S.-A., \& Casasanto, D. (2013). Space and time in the parietal cortex: fMRI evidence for a neural asymmetry. In Proceedings of the 35th Annual Meeting of the Cognitive Science Society (CogSci 2013), pp. 495-500.

Goodale, M. A., \& Milner, A. D. (1992). Separate visual pathways for perception and action. Trends in Neurosciences, 15, 20-25.

Halari, R., Sharma, T., Hines, M., Andrew, C., Simmons, A., \& Kumari, V. (2006). Comparable fMRI activity with differential behavioural performance on mental rotation and overt verbal fluency tasks in healthy men and women. Experimental Brain Research, 169, 1-14.

Hambrick, D. Z., Oswald, F. L., Darowski, E. S., Rench, T. A., \& Brou, R. (2010). Predictors of multitasking performance in a synthetic work paradigm. Applied Cognitive Psychology, 24, 1149-1167.

Heinzel, A., Hautzel, H., Poeppel, T. D., Boers, F., Beu, M., \& Mueller, H. W. (2008). Neural correlates of subliminal and supraliminal letter processing-An event-related fMRI study. Consciousness and Cognition, 17, 699-713.

Hsu, W. Y., Zanto, T. P., Anguera, J. A., Lin, Y. Y., \& Gazzaley, A. (2015). Delayed enhancement of multitasking performance: Effects of anodal transcranial direct current stimulation on the prefrontal cortex. Cortex, 69, 175-185.

Jordan, K., Heinze, H. J., Lutz, K., Kanowski, M., \& Jäncke, L. (2001). Cortical activations during the mental rotation of different visual objects. Neuroimage, 13, 143-152.

Kesner, R. P., \& Long, J. M. (1998). Parietal cortex and a spatial cognitive map. Psychobiology, 26, 162-166.

Konig, C. J., Buhner, M., \& Murling, G. (2005). Working memory, fluid intelligence, and attention are predictors of multitasking performance, but polychronicity and extraversion are not. Human Performance, 18, 243-266.

Kubik, V., Zimmermann, M., Del Missier, F., Frick, A., \& Mäntylä, T. (2018). Spatial ability incrementally explains multi- but not dualtasking: Further evidence for the spatiotemporal hypothesis. In Psychonomics International Meetings 2018, 4063. Amsterdam.

Kubik, V., Zimmermann, M., Del Missier, F., Frick, A., \& Mäntylä, T. (2019). Multi beyond dual: Multitasking costs reflect unique aspects of spatial ability. Manuscript in preparation.

Kübler, S., \& Schubert, T. (2017). P217 disrupting cognitive control in dual-task situations by transcranial magnetic stimulation of the lateral prefrontal cortex. Clinical Neurophysiology, 128, e119.

Lee, J., Ku, J., Han, K., Park, J., Lee, H., Kim, K. R., et al. (2013). rTMS over bilateral inferior parietal cortex induces decrement of spatial sustained attention. Frontiers in Human Neuroscience, 7, 26.

Logie, R. H., Cocchini, G., Delia Sala, S., \& Baddeley, A. D. (2004). Is there a specific executive capacity for dual task coordination? Evidence from Alzheimer's disease. Neuropsychology, 18, 504-513.

Logie, R. H., Trawley, S., \& Law, A. (2011). Multitasking: Multiple, domain-specific cognitive functions in a virtual environment. Memory \& Cognition, 39, 1561-1574.

Loukopoulos, L. D., Dismukes, R. K., \& Barshi, I. (2009). The multitasking myth bandling complexity in real-world operations (p. 188). Ashgate studies in human factors for flight operations. Surrey, UK: Ashgate. 
Mäntylä, T. (2013). Gender differences in multitasking reflect spatial ability. Psychological Science, 24, 514-520.

Mäntylä, T., Coni, V., Kubik, V., Todorov, I., \& Del Missier, F. (2017). Time takes space: Selective effects of multitasking on concurrent spatial processing. Cognitive Processing, 18, 229-235.

Medeiros-Ward, N., Watson, J. M., \& Strayer, D. L. (2015). On supertaskers and the neural basis of efficient multitasking. Psychonomic Bulletin \& Review, 22, 876-883.

Meyer, D. E., \& Kieras, D. E. (1997). A computational theory of executive cognitive processes and multiple-task performance: Part 1. Basic mechanisms. Psychological Review, 104, 3-65.

Milner, A. D., \& Goodale, M. A. (2008). Two visual systems re-viewed. Neuropsychologia, 46, 774-785.

Morgan, B., D’Mello, S., Abbott, R., Radvansky, G., Haass, M., \& Tamplin, A. (2013). Individual differences in multitasking ability and adaptability. Human Factors: The Journal of the Human Factors and Ergonomics Society, 55, 776-788.

Morillon, B., Kell, C. A., \& Giraud, A.-L. (2009). Three stages and four neural systems in time estimation. Journal of Neuroscience, 29, 14803-14811.

Nijboer, M., Borst, J., van Rijn, H., \& Taatgen, N. (2014). Single-task fMRI overlap predicts concurrent multitasking interference. Neuroimage, 100, 60-74.

Núñez, R., \& Cooperrider, K. (2013). The tangle of space and time in human cognition. Trends in Cognitive Sciences, 17, 220-229.

Oswald, F. L., Hambrick, D. Z., \& Jones, A. L. (2007). Keeping all the plates spinning: Understanding and predicting multitasking performance. In D. H. Jonassen (Ed.), Learning to solve complex scientific problems (pp. 77-97). Mahwah, NJ: Erlbaum.

Peters, M., Laeng, B., Latham, K., Jackson, M., Zaiyouna, R., \& Richardson, C. (1995). A redrawn Vandenberg and Kuse mental rotations test-Different versions and factors that affect performance. Brain and Cognition, 28, 39-58.

Pruim, R. H. R., Mennes, M., van Rooij, D., Llera, A., Buitelaar, J. K., \& Beckmann, C. F. (2015). ICA-AROMA: A robust ICAbased strategy for removing motion artifacts from fMRI data. Neuroimage, 112, 267-277.

Richter, W., Ugurbil, K., Georgopoulos, A., \& Kim, S. G. (1997) Time-resolved fMRI of mental rotation. NeuroReport, 8 , 3697-3702.

Risko, E. F., \& Gilbert, S. J. (2016). Cognitive offloading. Trends in Cognitive Sciences, 20, 676-688.

Sack, A. T. (2009). Parietal cortex and spatial cognition. Behavioural Brain Research, 202, 153-161.

Samrani, G., Marklund, P., Engström, L., Broman, D., \& Persson, J. (2018). Behavioral facilitation and increased brain responses from a high interference working memory context. Scientific Reports, 8, 15308.

Singh-Curry, V., \& Husain, M. (2009). The functional role of the inferior parietal lobe in the dorsal and ventral stream dichotomy. Neuropsychologia, 47, 1434-1448.

Sokolowski, H. M., Fias, W., Bosah Ononye, C., \& Ansari, D. (2017). Are numbers grounded in a general magnitude processing system? A functional neuroimaging meta-analysis. Neuropsychologia, 105, 50-69.

Stelzel, C., Kraft, A., Brandt, S. A., \& Schubert, T. (2008). Dissociable neural effects of task order control and task set maintenance during dual-task processing. Journal of Cognitive Neuroscience, 20, 613-628.

Strobach, T., Antonenko, D., Schindler, T., Flöel, A., \& Schubert, T. (2016). Modulation of executive control in the task switching paradigm with transcranial direct current stimulation (tDCS). Journal of Psychophysiology, 30, 55-65.

Strobach, T., Wendt, M., \& Janczyk, M. (2018). Editorial: Multitasking: Executive functioning in dual-task and task switching situations. Frontiers in Psychology, 9, 108.

Szameitat, A. J., Lepsien, J., von Cramon, D. Y., Sterr, A., \& Schubert, T. (2006). Task-order coordination in dual-task performance and the lateral prefrontal cortex: An eventrelated fMRI study. Psychological Research, 70, 541-552.

Thesen, T., McDonald, C. R., Carlson, C., Doyle, W., Cash, S., Sherfey, J., et al. (2012). Sequential then interactive processing of letters and words in the left fusiform gyrus. Nature Communications, 3, 1284.

Todorov, I., Del Missier, F., Konke, L. A., \& Mäntylä, T. (2015). Deadlines in space: Selective effects of coordinate spatial processing in multitasking. Memory \& Cognition, 43 , 1216-1228.

Todorov, I., Del Missier, F., \& Mäntylä, T. (2014). Age-related differences in multiple task monitoring. PLOS ONE, 9, e107619.

Todorov, I., Kubik, V., Carelli, M. G., Del Missier, F., \& Mäntylä, T. (2018). Spatial offloading in multiple task monitoring. Journal of Cognitive Psychology, 30, 230-241.

Tschernegg, M., Neuper, C., Schmidt, R., Wood, G., Kronbichler, M., Fazekas, F., et al. (2017). fMRI to probe sex-related differences in brain function with multitasking. PLOS ONE, 12, e0181554.

Tukey, J. W. (1977). Exploratory data analysis. Reading, MA: Addison-Wesley.

Vandenberg, S. G., \& Kuse, A. R. (1978). Mental rotations, a group test of three-dimensional spatial visualization. Perceptual and Motor Skills, 47, 599-604.

Verghese, A., Garner, K. G., Mattingley, J. B., \& Dux, P. E. (2016). Prefrontal cortex structure predicts training-induced improvements in multitasking performance. Journal of Neuroscience, 36, 2638-2645.

von Bastian, C. C., \& Druey, M. D. (2017). Shifting between mental sets: An individual differences approach to commonalities and differences of task switching components. Journal of Experimental Psychology: General, 146, 1266-1285.

Wager, T. D., \& Smith, E. E. (2003). Neuroimaging studies of working memory: A meta-analysis. Cognitive, Affective, $\varepsilon$ Behavioral Neuroscience, 3, 255-274.

Wickens, C. D. (2008). Multiple resources and mental workload. Human Factors: The Journal of the Human Factors and Ergonomics Society, 50, 449-455.

Wilhelm, O., Hildebrandt, A., \& Oberauer, K. (2013). What is working memory capacity, and how can we measure it? Frontiers in Psychology, 4, 433.

Wilson, A. D., \& Golonka, S. (2013). Embodied cognition is not what you think it is. Frontiers in Psychology, 4, 58.

Winkler, A. M., Ridgway, G. R., Douaud, G., Nichols, T. E., \& Smith, S. M. (2016). Faster permutation inference in brain imaging. Neuroimage, 141, 502-516.

Winkler, A. M., Ridgway, G. R., Webster, M. A., Smith, S. M., \& Nichols, T. E. (2014). Permutation inference for the general linear model. Neuroimage, 92, 381-397.

Worsley, K. J. (2001). Statistical analysis of activation images. In P. Jezzard, P. M. Matthews, \& S. M. Smith (Eds.), Functional magnetic resonance imaging (pp. 251-270). Oxford, United Kingdom: Oxford University Press.

Zacks, J. M. (2008). Neuroimaging studies of mental rotation: A meta-analysis and review. Journal of Cognitive Neuroscience, 20, $1-19$

Zhang, J., \& Norman, D. A. (1994). Representations in distributed cognitive tasks. Cognitive Science, 18, 87-122. 